\title{
ESTUDIO DEL CONFORT TÉRMICO Y LA CALIDAD CLIMÁTICA EN EL ESPACIO PÚBLICO. ESTUDIO DE CASO EN LA CIUDAD DE CHILLÁN, CHILE
}

\author{
Pamela SMITH GUERRA ${ }^{1}$, Cristián HENRÍQUEZ RUÍZ ${ }^{2}$ \\ ${ }^{1}$ Departamento de Geografia, Universidad de Chile. \\ ${ }^{2}$ Instituto de Geografia, Pontificia Universidad Católica de Chile. \\ pamelasmit@uchilefau.cl, autora@email.com
}

\section{RESUMEN}

En las últimas décadas, el estudio del clima en áreas urbanas ha venido despertando un interés creciente, en la medida que se han constatado problemas ambientales de las ciudades relacionados en mayor o menor grado, con los parámetros que lo definen. El estudio del clima urbano comprende diversas escalas de análisis espacial. Esta investigación abordó, en primer lugar, el estudio del Disconfort térmico a escala local en cuatro ciudades chilenas: Antofagasta, Viña del Mar, Chillán y Concepción, para luego profundizar en la micro escala a través del estudio del Confort Térmico Percibido en Espacios Públicos Exteriores seleccionados. Para la micro escala se escogió la ciudad de Chillán, debido a que concentra la mayor cantidad de horas diurnas expuestas a Disconfort térmico por calor en verano. Se realizaron encuestas de percepción durante una ola de calor ocurrida entre el 29 de enero y el 01 de febrero del año 2016. Los resultados expuestos corresponden a un avance de la investigación que a través de un enfoque metodológico mixto que combina metodologías cuantitativas que consideran datos instrumentales, y cualitativas, busca conocer cómo afecta el micro clima el uso de los espacios públicos, y evaluar también la calidad climática de estos espacios y el área urbana en general, entendiendo que ésta aporta a aumentar la calidad de vida en la ciudad.

Palabras clave: Clima urbano, Confort Térmico, Espacio público, Chillán.

\begin{abstract}
In recent decades, the study of climate in urban areas has been arousing increasing interest, to the extent they have found environmental problems of cities related to a greater or lesser degree, with the parameters that define it. The study of urban climate comprises different scales of spatial analysis. This research addressed, first, the study of thermal discomfort at the local level in four Chilean cities: Antofagasta, Vina del Mar, Chillán and Concepción, and then delve into the micro scale through the study of Thermal Comfort Perceived Foreign Public Spaces selected. For the micro scale Chillán was chosen because it has the largest number of daylight hours exposed to thermal discomfort in summer heat. perception surveys were conducted during a heat wave occurred between 29 January and 1 February 2016. The above results correspond to an advance of research through a mixed methodological approach that combines quantitative methodologies considered instrumental data, and qualitative, seeks to understand how it affects the micro climate the use of public spaces, and also evaluate
\end{abstract}


the climatic conditions of these spaces and urban areas in general, understanding that it brings to improve the quality of life in the city.

Key words: Urban climate, Thermal Comfort, Public Space, City of Chillán,

\section{INTRODUCCIÓN}

El acelerado proceso de urbanización experimentado en las últimas décadas a escala global se traduce en importantes alteraciones de origen antrópico asociadas al funcionamiento de la ciudad (Terradas et al., 2011). La modificación del ciclo hidrológico, la fragmentación de hábitats, la contaminación y formación de un clima urbano específico corresponden a algunos de los impactos socio-ambientales producidos por la urbanización, debido a su intensa transformación del medio natural. En las últimas décadas, el estudio del clima en áreas urbanas ha venido despertando un interés creciente, en la medida que se han constatado problemas ambientales relacionados en mayor o menor grado, con los parámetros que lo definen.

El clima urbano resulta de las modificaciones locales que las superficies, materiales y actividades de las áreas urbanas provocan en los balances de energía, masa y movimiento al reemplazar las condiciones naturales previas (Arnfield, 2003). Se entiende como una perturbación local del clima regional (Montávez et al., 2000) y se define por comparación de los rasgos climáticos al interior de la ciudad con aquellos presentes en su entorno rural próximo (Stewart \& Oke, 2009). Entre las características del clima urbano destacan la formación de islas de calor, sequedad, frío y humedad, las cuales pueden llegar a ser un factor de riesgo, favoreciendo la ocurrencia de enfermedades respiratorias, situaciones de disconfort y estrés térmico, entre otros.

El estudio del clima urbano comprende diversas escalas de análisis espacial. En cada una de ellas, el comportamiento de la temperatura atmosférica se explica por diferentes factores ambientales y urbanos, a partir de los cuales se han construido diversas clasificaciones. La presente investigación aborda la micro-escala. Las escalas regional (ciudad - entorno) y local (ciudad / intraurbana) se consideran como un marco que ayuda a describir el contexto y definir las articulaciones con la micro escala. La micro-escala corresponde a la escala de vecindad inmediata, abarca algunos cientos de metros y se desarrolla en el UCL (Urban Canopy Layer), que comprende el espacio ubicado entre el suelo y el límite superior de las edificaciones (Oke, 1987), esto es, el espacio donde las personas viven, actúan y sufren los agravios de los problemas de la ciudad (Gómez y Ferrer, 2010). La importancia de esta escala, desde el punto de vista climático, es que nos permite comprender la relación que se establece entre la sociedad y las condiciones micro meteorológicas, evaluar el bienestar térmico del peatón y entender el nexo entre la morfología urbana y el microclima, aportando insumos a la planificación sustentable de la ciudad (Cárdenas, 2012).

El hombre tiene necesidad de mantener su organismo a una temperatura constante en torno a un valor de $37^{\circ} \mathrm{C}$. La temperatura corporal puede verse afectada por las condiciones ambientales del entorno y aun cuando esto no signifique un riesgo para la salud, sí puede incidir en el nivel de satisfacción de las personas y en consecuencia, en su experiencia, modificando el uso y permanencia en un lugar expuesto a condiciones poco favorables. 
De acuerdo a Nikolopoulou et al. (2001), hoy existe un gran interés por conocer el confort térmico en espacios públicos exteriores, considerando que estos pueden contribuir a la calidad de vida de las ciudades y a disminuir el aislamiento y la exclusión social.

El concepto de confort climático es fundamental en la planificación de una ciudad, dado que la modificación de las condiciones climáticas que esta produce afecta la calidad de vida urbana, así como la percepción de las personas respecto del espacio público exterior y el uso que dan al mismo. En asociación al clima han surgido conceptos como el de justicia climática, para referirse a la distribución desigual de las consecuencias de la modificación climática al interior de la ciudad.

La presente investigación aborda el estudio de espacios públicos seleccionados en la ciudad de Chillán. En primer lugar se seleccionaron cuatro ciudades, las ciudades costeras de Antofagasta y Viña del Mar, ubicadas a la latitud $23^{\circ} 39^{\prime} \mathrm{S}$ y $33^{\circ} 02^{\prime} \mathrm{S}$ respectivamente, y luego, las ciudades de Chillán y Concepción, ubicadas a similar latitud ( $36^{\circ} 36^{\prime} \mathrm{S}$ y $\left.36^{\circ} 49^{\prime} \mathrm{S}\right)$, la primera, en el interior y la segunda en la costa. Del estudio de las horas diurnas con Disconfort Térmico se seleccionó una de estas. Como fin último se busca determinar el modo en que el microclima afecta el uso de los espacios públicos, a partir del estudio del confort instrumental y percibido, así como evaluar la calidad climática de estos espacios con el fin de proponer lineamientos de planificación y diseño urbano que consideren el micro clima en la planificación de los mismos, como un aporte al mejoramiento de la calidad de vida en la ciudad. Se presenta un avance de los resultados asociados a la medición del Confort Térmico percibido en verano por los usuarios de los espacios públicos seleccionados.

\section{MÉTODOS}

La investigación se desarrolla a través de una aproximación metodológica mixta, que combina métodos de naturaleza cualitativa y cuantitativa. Esta etapa de la investigación presenta un enfoque principalmente cuantitativo. La información cuantitativa es generada a partir de fuentes secundarias pre-existentes, del procesamiento de imágenes satelitales y del levantamiento de datos en terreno, a través de la observación y medición instrumental.

En primer lugar se caracterizan las ciudades escogidas a partir del análisis de las horas estudio del confort térmico a escala local considerando la propuesta de Zonas de Confort de Olgyay (1967) a partir del comportamiento de la temperatura atmosférica y humedad relativa del aire. Olgyay propuso una zona de confort que se encontraba entre $\operatorname{los} 21,1$ y $26,7^{\circ} \mathrm{C}$ para una humedad relativa que oscila entre 20 y $80 \%$. Lo anterior fue definido para la latitud $40^{\circ}$ para un arropamiento de 1 clo, que corresponde a una unidad arbitraria para determinar el grado de aislamiento de una prenda de vestir y un índice metabólico de 1,5 que corresponde a un paseo ligero. El autor plantea una metodología de adaptación basada en la localización de las ciudades, indicando que por cada $5^{\circ}$ menos de latitud, debían sumarse $0,4^{\circ} \mathrm{C}$ al límite superior de la zona de confort. Se identifican las horas expuestas a condiciones de confort y disconfort térmico en la estación de primavera y verano de acuerdo a los límites indicados en la tabla 1 . 


\begin{tabular}{|cccccc|}
\hline Ciudad & Latitud & $\begin{array}{c}\text { Dif. con } \\
\text { Olgyay }\left({ }^{\circ}\right)\end{array}$ & $\begin{array}{c}\text { Aumento límite } \\
\text { superio }\left({ }^{\circ} \mathrm{C}\right)\end{array}$ & $\begin{array}{c}\text { Límite } \\
\text { superior }\end{array}$ & $\begin{array}{c}\text { Límite } \\
\text { Inferior }\end{array}$ \\
\hline Antofagasta & 23 & 13 & 1,04 & 27,74 & 21,1 \\
\hline Viña del Mar & 33 & 7 & 0,56 & 27,26 & 21,1 \\
\hline Chillán & 36 & 4 & 0,32 & 27,02 & 21,1 \\
\hline Concepción & 36 & 4 & 0,32 & 27,02 & 21,1 \\
\hline
\end{tabular}

Tabla 1. Limites de la zona de confort de las ciudades estudiadas.

El Confort Térmico Percibido se midió a partir de la realización de encuestas en terreno entre el 29 de enero y 01 de febrero de 2016. Se consultaron usuarios de los espacios públicos, mayores de 18 años, seleccionados al azar. La encuesta incluyo cuatro dimensiones: 1. Descripción general del encuestado, 2. Percepción del clima, 3. Análisis Espacial, 4. Uso del Espacio. Dentro de la percepción del clima se evaluó el Confort General del a través de la pregunta ¿Cómo se siente respecto de la temperatura? que era contestada considerando una escala de 11 puntos, que va de 0 a 10 , los valores de 0 a 3 se asocian con una situación de Frío, muy inconfortable, de 4 a 6 , el estado general era bueno, y de 7 a 10, el individuo sentía Calor, muy inconfortable basados en la escala utilizada por Lamarca (2014).

\section{RESULTADOS}

\subsection{Confort térmico a escala local}

Para el cálculo de confort térmico de la ciudad se escogieron estaciones meteorológicas representativas de las áreas urbanas, localizadas en áreas construidas cercanas al centro de cada una de las ciudades. El gráfico 1 muestra como se distribuyen las horas diurnas (entre las 8 am y 10 pm) durante el año, classificadas en situaciones de Confort y Disconfort Térmico, este último associado a Disconfort por calor. Las horas restantes corresponden a aquellas cuya temperatura atmosférica se encuentra bajo el limite inferior de la zona de confort definida en la tabla 1.

La ciudad que presenta un mayor número de horas de disconfort térmico diurno es Chillán, destacando la proporción de horas de disconfort por calor concentradas principalmente en verano. Las ciudades de Concepción y Viña del Mar registran una importante proporción de horas con disconfort por frío, con temperaturas que se encuentran por debajo del límite inferior de $21,1^{\circ}$. Antofagasta, pese a su latitud, concentra el menor número de horas en confort térmico, lo que puede deberse en parte a su ubicación costera, pero además a las bondades de la orientación que predomina en su trama urbana que favorece la entrada y circulación de los vientos de mar a tierra durante el día.

La mayor cantidad de horas asociadas a Disconfort térmico se presentan en la estación de verano, distrinbuidas durante el día con un pic cercano a las 16:00 hrs.

Por lo anteriormente expuesto se analiza más profundamente la ciudad de Chillán y se escoge además un día de verano promedio para espacializar las zonas de confort y disconfort térmico en la ciudad a partir de la interpolación de temperaturas a las 16:00 hrs. 


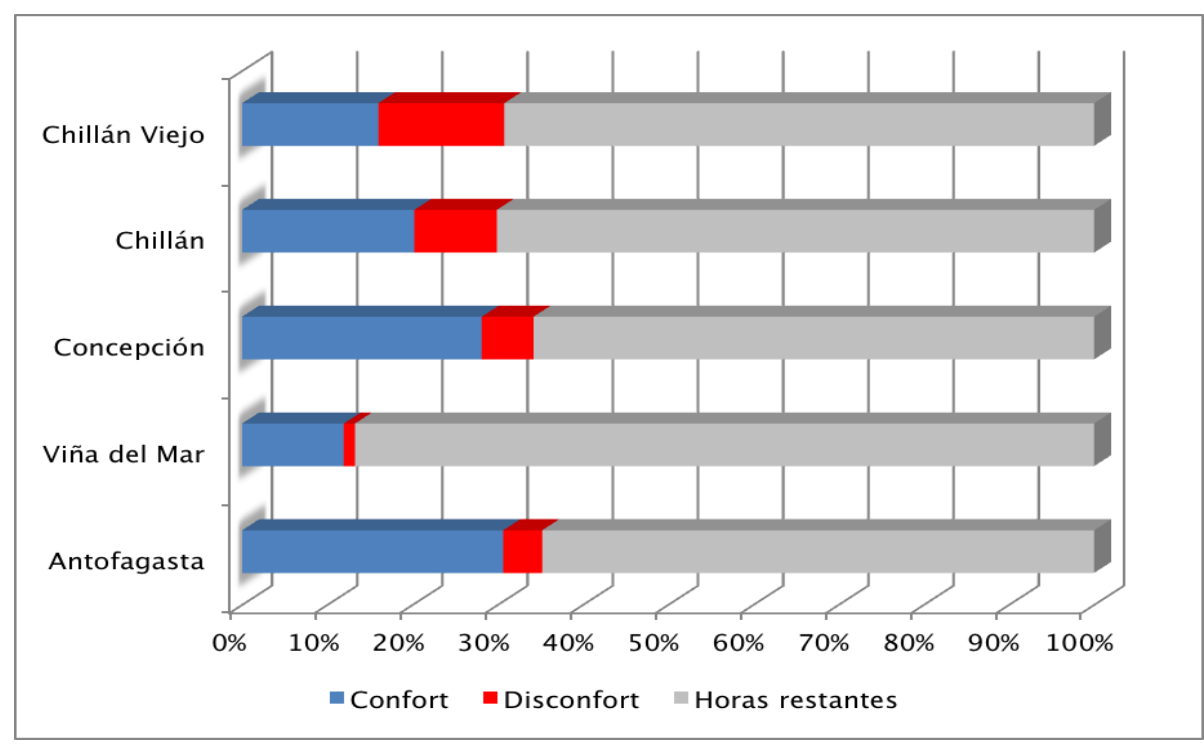

Gráfico 1. Horas de Confort y Disconfort Térmico en las ciudades estudiadas.

Fuente: Elaboración propia.

\subsection{Confort térmico a micro escala}

Al interior de las comunas de Chillán y Chillán Viejo se escogieron cinco espacios públicos:

- La plaza de Armas de Chillán (nº1 de la figura 1)

- El Paseo Peatonal Arauco (n² de la figura 1)

- El Parque Estero Las Toscas (n³ de la figura 1)

- Parque Sara Gajardo (nº de la figura 1)

- Parque Monumental Bernardo O’Higgins (n5 de la figura 1)

Durante la campaña de terreno del verano de 2016 se realizaron un total de 362 encuestas. Del total de encuestados, $45,5 \%$ (143) son mujeres y $54,5 \%$ (171) son hombres. Esta proporción se mantiene para todos los espacios públicos considerados. La mayor proporción de mujeres se observa en el Paseo Peatonal Arauco con 47.7\% y la menor proporción en Estero Las Toscas representando el 42. La mayor cantidad de encuestados tiene entre 21 y 30 años. El 95\% de los encuestados se encuentra entre los 18 y 60 años. La mayoría corresponde a trabajadores dependientes o estudiantes.

En términos generales, considerando de manera agregada todos los espacios públicos estudiados, las categorías asociadas al calor, muy inconfortable dominan representando un $76 \%$ del total de respuestas, el $24 \%$ restante se encontraba en una situación de confort térmico. Solo un encuestado ubicado en la Plaza de Armas de Chillán declaro sentirse en una situación muy inconfortable debido al frío a las 18:35 hrs. del día viernes 29 de enero. No se registran diferencias significativas asociadas al sexo de los encuestados. 
Si se observa lo anterior por espacio público, prevalece el disconfort por calor (más del 80\% de los encuestados) en la Plaza de Armas de Chillán, el Paseo Peatonal Arauco y el Parque Monumental Bernardo O’Higgins de Chillán Viejo, todos ubicados en las zonas más cálidas de ambas comunas. Los espacios públicos en que la percepción de confort térmico alcanza el 30\% son Parque Estero Las Toscas y Sara Gajardo.
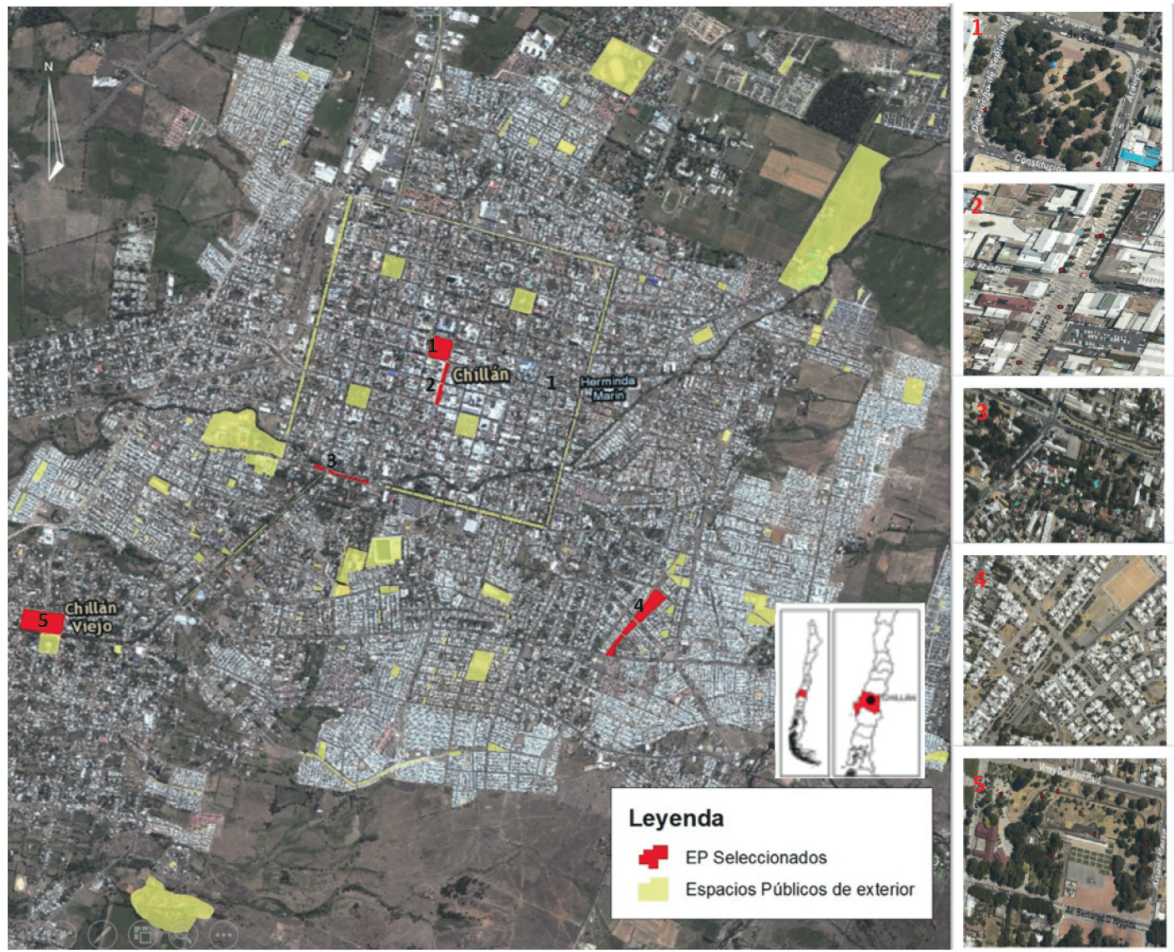

Figura 1. Espacios públicos seleccionados en la ciudad de Chillán.

Fuente: Elaboración propia.

Considerando la naturaleza compleja del concepto de confort térmico, su análisis puede enriquecerse incorporando categorías de análisis subjetivas como por ejemplo el nivel de expectativas en torno al comportamiento de la temperatura (Lenzholzer et al., 2015), las expectativas son dinámicas y van fijando nuevos límites de resistencia de los individuos. Estas pueden estar influidas por la experiencia al largo, mediano o incluso el corto plazo, por el lugar de origen, de residencia, la estación del año, el comportamiento del tiempo los días u horas previas, o el motivo de uso del espacio público.

En un primer intento por relacionar el confort térmico con las expectativas se cruzó el resultado de la encuesta con los motivos de uso, agrupados en ocho categorías como se muestra en el gráfico número dos. Para la interpretación del gráfico se debe recordar que los valores superiores a 7 se asocian a disconfort por calor ("calor, muy 
insoportable") y que el confort térmico se encuentra entre los valores 5 y 6 . Si bien, en la mayoría de los casos el disconfort se asocia a más del $80 \%$ de las respuestas, existen algunas excepciones tales como el relajarse y descansar, o la recreación en que la importancia relativa de quienes se encuentran en situación de confort térmico aumenta, alcanzando el 20\%. Lo anterior solo es superado por quienes declaran estar trabajando o realizando trámites en el espacio público, en este caso los encuestados se asocian principalmente al Paseo Peatonal Arauco que se configura como un cañón urbano de dos cuadras, con presencia de árboles en ambos costados, sin circulación vehicular y donde los usuarios se encuentran en movimiento, de pasada, sin permanecer largos períodos en el espacio público.

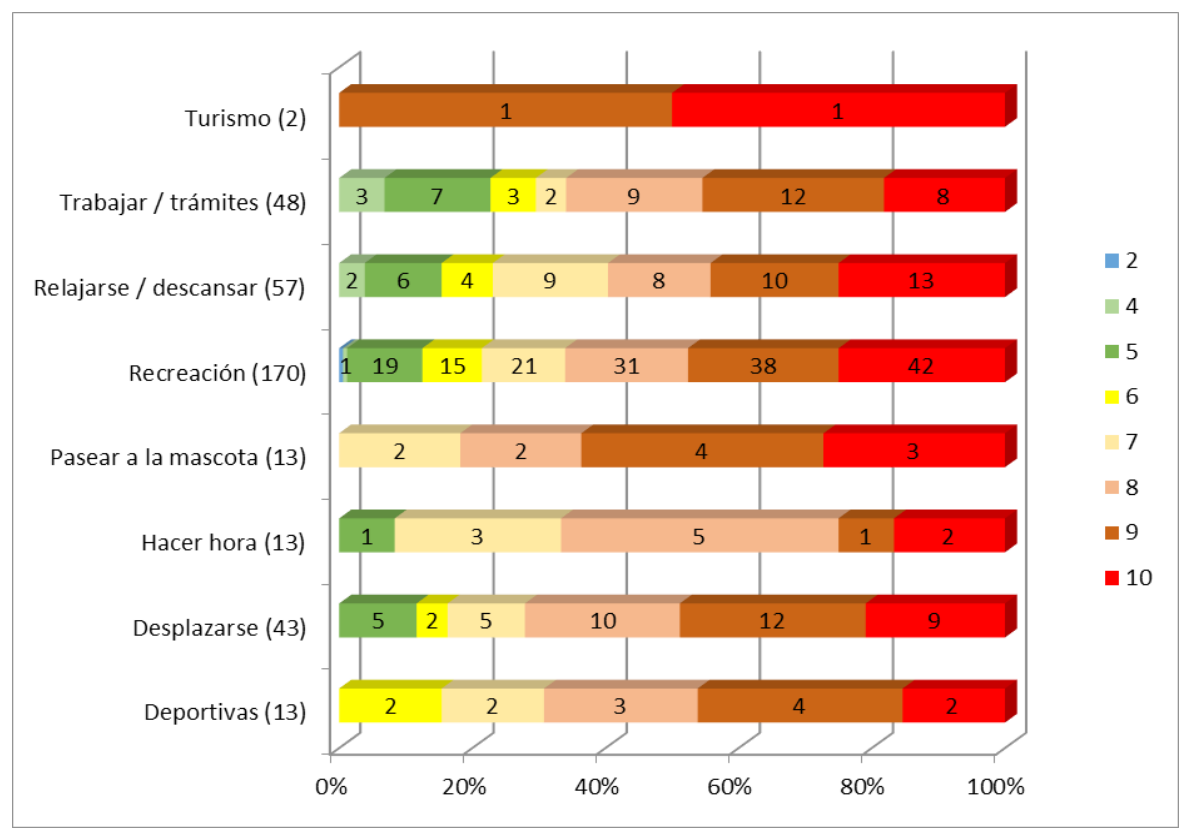

Gráfico 2. Percepción de Confort y Disconfort Térmico según motivo de uso. Fuente: Elaboración propia.

La mayoría de los usuarios encuestados en los espacios públicos declara que existen diferencias en el uso de estos en las diferentes estaciones del año. El gráfico tres muestra las principales razones que explican por espacio público las diferencias en el uso. Destacan las razones que se asocian al clima, como por ejemplo el calor, el frío o la lluvia. En algunos espacios públicos como el Parque Bernardo O'Higgins alrededor del $90 \%$ de los encuestados señalo al clima como el responsable de la disminución del uso en invierno por el frío y la lluvia y el aumento en verano por las "mejores condiciones climáticas" principalmente asociadas a mayores temperaturas. Entre las razones de otra índole que explican la disminución en la frecuencia de uso de los espacios públicos destaca la disminución del tiempo libre, la disminución de las horas de luz, menos usuarios y menos actividades, sobre todo en la estación de invierno. 


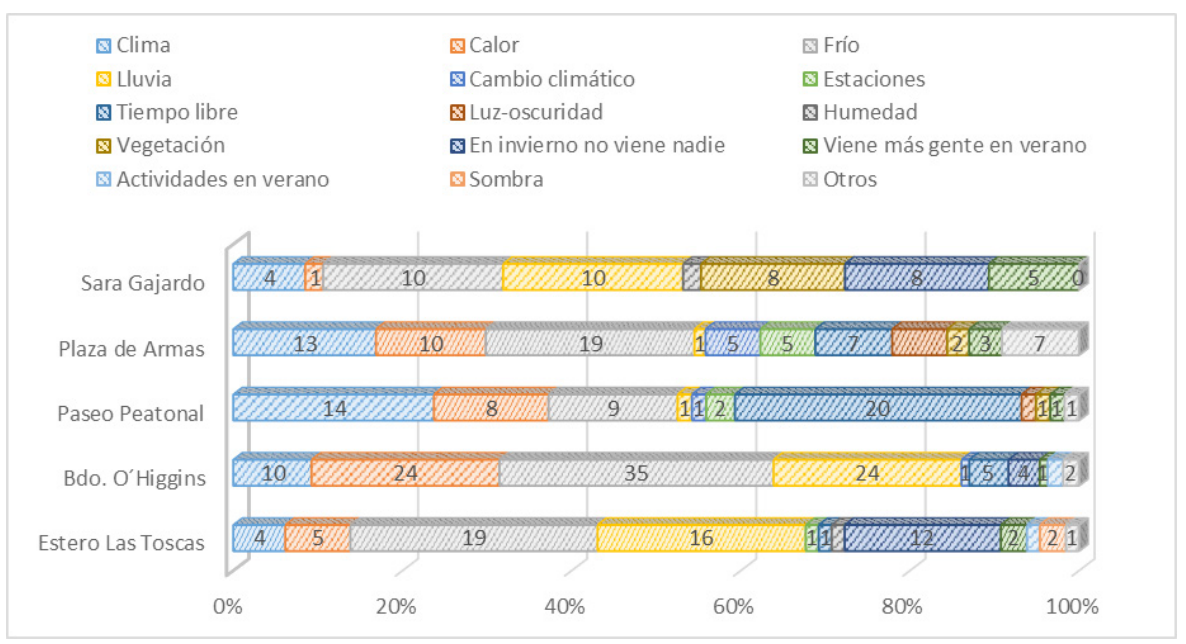

Gráfico 3. Razones que explican la modificación del uso de los espacios públicos durante el año. Fuente: Elaboración propia.

\section{DISCUSIÓN}

Pese a su latitud, a escala local la ciudad con mayor disconfort térmico es Chillán, y esto se demuestra tanto por el número de horas al año asociadas a esta condición, como al observar espacialmente la distribución, con un gran número de espacios públicos afectados por disconfort por calor durante la tarde de un día promedio, y más aún, durante la tarde del día más caluroso del verano. Por otra parte, la ciudad de Antofagasta, aun cuando corresponde a la ciudad más septentrional estudiada, registra el mayor número de horas asociada a confort térmico, principalmente en verano y en las estaciones intermedias, primavera y otoño. Lo que puede deberse en parte a la orientación de la trama urbana, al menos en su parte fundacional, que permite la entrada de los vientos desde el mar, generando una mayor ventilación de la ciudad.

El estudio del Confort Térmico percibido permite el análisis desde variados puntos de vista, en primer lugar se realizo una descripción general de los resultados que dan cuenta de la situación de insatisfacción que perciben los usuarios de los espacios públicos seleccionados en la ciudad de Chillán en las tardes de verano que además coinciden con la existencia de una ola de calor que comienza el segundo día de terreno, el sábado 30 de enero.

De acuerdo a la literatura, las expectativas constituyen un asunto importante a la hora de evaluar el confort térmico percibido, en este sentido, el cruce de este con los motivos de uso marca una sutil tendencia, sin embargo los resultados encontrados para quienes declaran trabajar o realizar trámites llaman la atención y podrían estar relacionados con la naturaleza del espacio público en que principalmente se concentran, el Paseo Peatonal en que el uso es en movimiento.

Se espera profundizar en lo anterior y en otros aspectos que permitan ir dando cuenta de la importancia de las expectativas en el confort, sobre todo aquellas que se 
relacionan con factores geográficos como el lugar de residencia de los usuarios que podría ser un indicador de la existencia de injusticia climática urbana.

\section{AGRADECIMIENTOS}

Esta investigación se encuentra financiada por el Proyecto de Investigación FONDECYT 1130305 "Estudio y modelación del clima urbano a escala local, como base para la proposición de lineamientos de adaptación frente al cambio climático en una red de ciudades Chilenas".

Se agradece a: Marianela Baeza, Valentina Durán, Matías González, Ma . Alejandra Moreno, Paulo Moreno, Carolina San Martín, Braian Villarreal y Camila Yáñez que participaron en la realización de encuestas y monitoreo de parámetros meteorológicos durante la campaña de terreno realizada en los meses de enero y febrero del año 2016.

\section{REFERENCIAS}

Arnfield, J. (2003). Two decades of urban climate research: A review of turbulence, exchanges of energy and water, and the urban heat island. International Jornal of Climatology 23 (1), $1-26$.

Cárdenas, L. (2012). Caracterización de patrones bioclimáticos en tejidos urbanos residenciales. Cuadernos de Investigación Urbanística 82 (1), 1 - 71.

Gómez, N. and Ferrer, M. (2010). Microclimatic study of the space between buildings. Case: Urban housing developments, Maracaibo (Venezuela), Rev. Téc. Ing. Univ. Zulia. 33 (3), 223 - 234.

Lamarca, C. (2014). Comparación de modelos físicos y perceptuales para determinar el confort térmico en distintos cañones urbanos de la ciudad de concepción. (Tesis de Master. Director: Dr. Cristian Henríquez, Pontificia Universidad Católica de Chile. Instituto de Geografía, Santiago de Chile).

Lenzholzer, S.; Klemm, W. \& Vasilikou, C. (2015). New qualitative methods to explore termal perception in urban spaces. Conference proceedings ICUC9 - 9th International Concference on Urban Climate jointly with 12th Symposium on the Urban Environment, Julio 2015.

Montávez, J; Rodríguez, A \& Jimenez, J. A (2000). study of the urban heat island of Granada. International Journal of Climatology 20 (1), 899 - 911.

Nikolopoulou, M.; Baker, N. and Steemers, K. (2001). Thermal comfort in outdoor urban spaces: understanding the human parameter. Solar Energy 70 (3), 227-235.

Olgyay, V. (1963). Arquitectura y Clima: Manual de diseño bioclimático para arquitectos y urbanistas, Editorial Gustavo Gili: Barcelona.

Stewart, I and Oke, T. (2009). Classifying urban climate field sites by local climate zones: The case of Nagano japan. Seventh International Conference or Urban Climate. 29 June - 3 July. Yokohama. Japan, 1 - 5.

Terradas, J. (2001). Ecología Urbana. Barcelona, Rubes Editorial, S. L. Tobler. Terradas, J.; Franquesa, T.; Parés; M. y Chaparro, L. . En Ecología Urbana. Investigación y Ciencia, $52-60$. 